\title{
Predicting road traffic density using a machine learning-driven approach
}

\author{
Abdelhafid Zeroual* $^{*}$, Fouzi Harrou ${ }^{\S}$, Ying Sun ${ }^{\S}$ \\ Department of Whatever,Faculty of technology, University of 20 August 1955, Skikda 21000, Algeria *, \\ Computer, Electrical and Mathematical Sciences and Engineering (CEMSE) Division, \\ King Abdullah University of Science and Technology (KAUST), Thuwal 23955-6900, Saudi Arabia $\S$ \\ Email: *a.zeroual@univ-skikda.dz, ${ }^{\S}$ fouzi.harrou@kaust.edu.sa
}

\begin{abstract}
Notwithstanding the technological developments in transportation systems, traffic congestion is still hampering the growth and development of countries. Accurate traffic flow prediction plays an essential role in intelligent transportation systems to mitigate traffic congestion problems. Importantly, it provides prior knowledge on traffic status, which enables avoiding congested points. This paper employed a Support vector regression (SVR) approach, a kernel-based learning model, to predict traffic flow. We assessed the efficiency of the SVR model for traffic density prediction by considering different types of kernels. We used traffic data from California highways to test the SVR prediction performance. Results showed that SVR with Gaussian kernel dominates the other SVR models.
\end{abstract}

Index Terms-ITS, Traffic flow prediction, Machine learning, SVR.

\section{INTRODUCTION}

Precise traffic flow information is crucial in developing intelligent transportation systems (ITSs) and guaranteeing effective management of traffic flow [1], [2]. Predicting traffic flow represents a necessary step to mitigate traffic congestion and provide important information to individual travelers and different government sectors. Essentially, traffic flow prediction aims to provide pertinent information to help road users optimally deal with traffic congestion by using more appropriate travel routes, evaluating roadway safety, reducing pollution, and enhancing traffic operation efficacy [3], [4]. Hence, developing an effective data-driven approach to predict traffic flow is our main objective in this study.

Over the last years, considerable development in motorway and transportation technology has been witnessed. Accordingly, a significant consideration in terms of traffic management and control is needed to avoid traffic congestion and accidents. Intelligent transportation systems come up with many alternative solutions and strategies to remedy traffic challenges [5]. Traffic density, one of the most important traffic parameters in the transportation system that reflected the evolution of congestion phenomena in its several statuses from traffic release to bottling, can be used as an indicator for traffic management.
Traffic flow is characterized by a nonlinear structure, making its prediction using physical approaches more difficult and complex. Various methods have been designed in the literature to predict traffic density, including model-based and data meaning-based approaches [6], [7]. Model-driven approaches are based on mathematical models that describe several physical phenomena. Numerous analytical methods are developed in the literature, including microscopic and macroscopic models. Unlike model-based techniques, where prior availability of an analytical model describing the traffic dynamics is required, only historical traffic measurement is needed in data-driven techniques [8].

With the advanced computation technologies and the availability of large data in transportation systems, predicting traffic flow with high accuracy and more realistic via intelligent systems and machine learning approaches is necessary [9]. Several data-driven methods have been proposed for traffic prediction. For instance, in [10], an Autoregressive Integrated Moving Average (ARIMA) is considered for traffic prediction. Similarly, in [11], seasonal ARIMA is applied to forecast traffic data using stationary transformation and the optimal Bayesian criterion. Authors in [12] introduced a method using multivariate spatial-temporal autoregressive to predict both speed and volume traffic parameters. Other researchers focused on employing machine learning techniques such as neural network and decision trees methods to improve traffic flow prediction [13], [14]. Of course, machine learning models are non-parametric and flexible to model traffic flow data without an analytical model formulation.

This paper aims to employ a data-driven approach for improving traffic density prediction. Specifically, support vector regression (SVR), a kernel-based machine learning approach, is used in this study because of its suitable performance in nonlinear approximation. Crucially, SVR models exhibited high abilities for nonlinear system modeling with high accuracy [15]. In this study, the SVR model was assessed using traffic data from California highways. Results revealed that SVR with Gaussian kernel dominates the SVR models with Linear and 
quadratic kernels. The remainder of this paper is outlined as follows. In the next section, we briefly present the concept of the SVR model. In section III, we present the SVR traffic prediction framework process and the description of data used to validate the proposed prediction approach. Then, in section IV, we discuss the obtained results. Finally, we summarize and conclude this paper in section IV.

\section{SUPPORT VECTOR REGRESSION (SVR) APPROACH}

We present in this section the basic concept of the SVR model, a well-known kernel learning-based approach [16][18]. SVR models showed a high capacity to learn and model process nonlinearities through kernel tricks without any data distribution specification. In addition, SVR could provide satisfactory modeling performance in the presence of limited samples [17]. They showed to be useful in several applications as renewable energies modeling, and prediction [19], [20], State-of-charge prediction of battery [21], swarm motion prediction [22]. Importantly, to model input-output data, the SVR model adjusts the error inside a certain threshold instead of minimizing it like in classical regression models. The SVR model can be given by:

$$
f(x)=\mathbf{w} \cdot \phi(\mathbf{x})+b,
$$

Here, $f(x)$ refers to the function describing the link between the inputs and outputs variables, which is defined according to its close as smooth as possible to the actual data $y_{i}$ through $\epsilon$ for all training data [17]. Here, $\epsilon$ is the insensitive region of $f(x)$ (Figure 1) and which known by tube [23]. The occurred errors were considered to be reasonable if they were within the tube. However, the outside ones of are not acceptable. Considering a variable $y$ and a predictor $x$, the SVR outlines inputs $\left(\mathbf{x}_{i} \in \mathbb{R}^{n}\right)$ under multidimensional space of $n$ dimensions, which is known by feature space (1-dimensional SVR example can be shown in Figure 1).

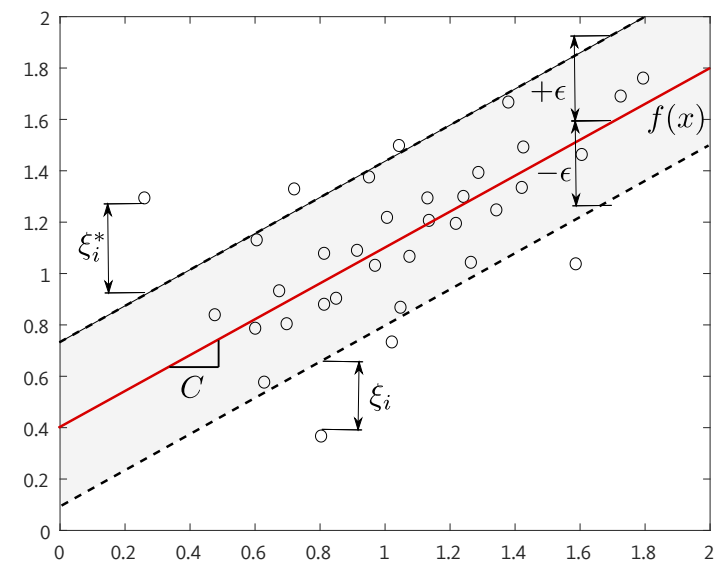

Fig. 1: 1-dimensional SVR mapping example

In short, SVR is performed by projecting the data to feature space via the non-linear mapping function $\phi$ and applying the linear regression. The weight vector, $\mathbf{w}$, and the bias, $b$, are determined by minimizing the convex quadratic problem [17]:

$$
\begin{gathered}
\min _{w, \xi, \xi^{*}}\left\{\frac{1}{2} w^{T} w+C \sum_{i=1}^{l}\left(\xi_{i}+\xi_{i}^{*}\right)\right\} \\
\text { under constraints }\left\{\begin{array}{l}
y_{i}-\left(w^{T} \phi\left(x_{i}\right)-b\right) \leq \epsilon+\xi_{i}, \\
w^{T} \phi\left(x_{i}\right)+b-y_{i} \leq \epsilon+\xi_{i}^{*}, \\
\xi_{i}, \xi_{i}^{*} \geq 0, i=1, \ldots, l .
\end{array}\right.
\end{gathered}
$$

Here, the constant $C>0$ permits managing the trade-off between error and tube. The slack variables, $\xi$ and $\xi^{*}$ allow the measurement between the point and the tube borders. These slack variables can be added to avoid outliers and provide some tolerated points, as shown in Figure 1. In addition, the slack variables are used to determine the error level. The equation (2) can be expressed as [17],

$$
\max \left\{\begin{array}{l}
\frac{1}{2} \sum_{i, j=1}^{m}\left(\alpha_{i}-\alpha_{i}^{*}\right)\left(\alpha_{j}-\alpha_{j}^{*}\right) K\left(x_{i}, x_{j}\right) \\
-\epsilon \sum_{i=1}^{m}\left(\alpha_{i}+\alpha_{i}^{*}\right)+\sum_{i=1}^{m} y_{i}\left(\alpha_{i}-\alpha_{i}^{*}\right)
\end{array}\right.
$$

where $\sum_{m}^{i=1}\left(\alpha_{i}-\alpha_{i}^{*}\right)=0,0 \leq \alpha_{i}, \alpha_{i}^{*} \leq C$. With $\alpha_{i}$ and $\alpha_{i}^{*}$ denotes the Lagrange multipliers [17]. The formulation (1) can be written as follows by using the kernel function:

$$
f(x)=\sum_{i=1}^{N}\left(\alpha_{i}-\alpha_{i}^{*}\right) k\left(x_{i}, x_{j}\right)+b,
$$

The kernel function $K\left(x_{i}, x_{j}\right) \equiv \phi\left(x_{i}\right)^{T} \phi\left(x_{j}\right)$ projects the inputs data into large dimensional space. This transformation permits mapping the input variables in feature space and provides high flexibility and modeling process nonlinearities. The radial basis function (RBF) kernel is one of the commonly used kernels, which is given as:

$$
K\left(x_{i}, x_{j}\right)=\exp \left(\frac{\left\|x_{i}-x_{j}\right\|^{2}}{2 \sigma^{2}}\right),
$$

where $\sigma$ refers to the width of Gaussian kernel.

\section{SVR TRAFFIC PREDICTION FRAMEWORK AND DATA DESCRIPTION}

\section{A. SVR traffic prediction framwork}

This paper proposes a machine learning-based approach to predict traffic densities. Figure 2 schematically presents the main steps of the prediction procedure. At first, traffic data (e.g., flow and speed) is collected via sensors from the monitored road section. Then, as the traffic density data cannot be provided directly from the traffic data collection system, it can be computed as follow:

$$
\text { Density }=\frac{\text { flow }}{\text { speed }} \text {. }
$$

Then, the obtained traffic density data is used to input the SVR model for traffic prediction. Specifically, the data is divided into two sets, training, and testing. We construct the SVR model using the training data. In training, the values of SVR model parameters are computed so that the prediction error is minimized. After, the trained model is used for traffic density prediction. 


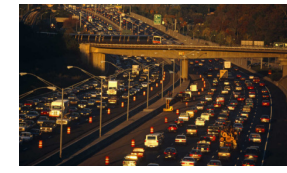

Traffic system

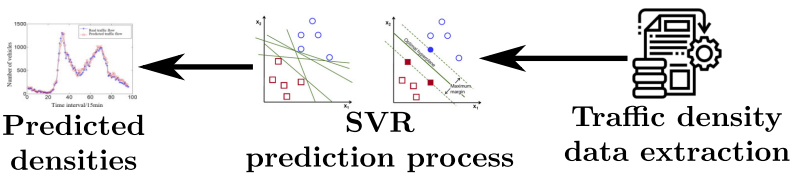

Fig. 2: SVR-based traffic density prediction framework.

\section{B. Data description}

To validate the proposed approach, we chose a public available traffic dataset from a road part in the highways of California. This dataset is collected from the Ashby Ave off-e-diag highway part, which is located in the est of the interstate 80 (Figure 3). This interstate is known as Dwight D. Eisenhower Highway and Blue Star Memorial Highway; it stretches from California to New Jersey, coming from San Francisco and ending to Teaneck.

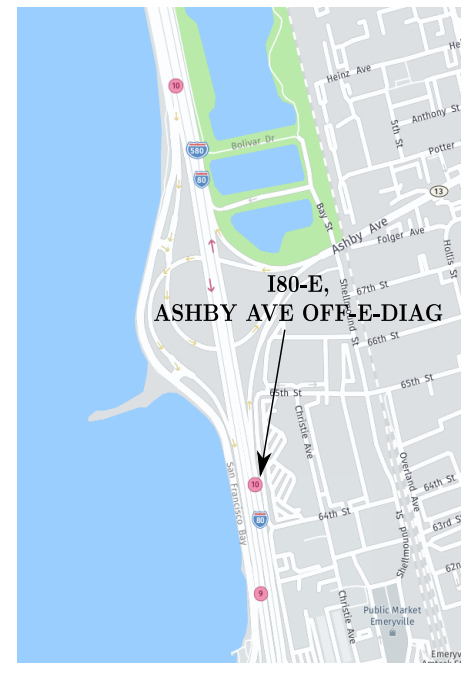

Fig. 3: Map of Ashby Ave off-e-diag highway part.

The herein collected data are for a period of seven days (from 18th December 2018 to 22th December 2018) with a step time of 5 minutes, as well as, these days contain various changing of traffic statuses.

\section{TRAFFIC DENSITY PREDICTION RESULTS}

\section{A. Performance validation metrics}

To assess the prediction accuracy of the SVR models, in this paper, we use four statistical scores: $R^{2}$, root-meansquare error (RMSE), mean absolute percent error (MAPE), and Mean Absolute Error (MAE). These metrics are obtained as follow:

$$
R^{2}=1-\frac{\sum_{k=1}^{n}\left(\rho_{k}-\hat{\rho}_{k}\right)^{2}}{\sum_{k=1}^{n}\left(\rho_{k}-\bar{\rho}_{k}\right)^{2}}
$$

$$
\begin{gathered}
R M S E=\sqrt{\frac{1}{n} \sum_{k=1}^{n}\left(\rho_{k}-\hat{\rho}_{k}\right)^{2}} \\
M A P E=\frac{1}{n} \sum_{k=1}^{n}\left|\frac{\rho_{k}-\hat{\rho}_{k}}{\rho_{k}}\right| \times 100 \\
M A E=\frac{1}{n} \sum_{k=1}^{n}\left|\rho_{k}-\hat{\rho}_{k}\right|
\end{gathered}
$$

where $n$ represents the data length, $\rho_{k}$ and $\hat{\rho}_{k}$ are the actual and predicted densities, and $\bar{\rho}_{k}$ is the mean value of actual densities. An accurate prediction is characterized by an $R^{2}$ value closer to 1 and lower values of other metrics.

\section{B. Prediction results and discussions}

In this part, to test and validate the SVR-based prediction approach, we first train the model using training, which comprises five days of traffic data. Then, we evaluate the model based on testing data.

Figure 4 (a-d) depicts the prediction results of the linear regression model and three SVR models (i.e., linear SVR prediction, quadratic SVR prediction, and Gaussian SVR) using training data. Figure 5 shows the scatter plot of the measured vs. predicted traffic density from the four investigated models. Visually, we observe that the SVR with Gaussian kernel fit well the training data and provided a better description of the traffic density than the other models.

Table I lists the accuracy metrics, which reflect the quality of the prediction proposed method for training and testing data. The gaussian SVR model dominates the other models by getting the higher $R^{2}$ and lowest RMSE and MAE values. Table I revealed that the linear models (linear regression and linear SVR) provide poor prediction performance. On the other hand, the nonlinear SVR models can model important variations in traffic density data.

TABLE I: Models evaluation based on R2, RMSE and MAE metrics.

\begin{tabular}{l|l|l|l}
\hline Models & R2 & RMSE & MAE \\
\hline Linear Regression & 0.58 & 97.255 & 70.32 \\
Linear SVR & 0.48 & 109.14 & 62.627 \\
Quadratic SVR & 0.92 & 42.115 & 29.19 \\
Gaussian SVR & 0.94 & 36.568 & 24.485 \\
\hline
\end{tabular}

Figure 6 shows the prediction results of the trained models based on testing data. It can be seen from Figure 6 that the Gaussian SVR can more accurately predict the evolution of the traffic density.

Figure 7 shows the distribution of prediction errors of the four methods using the test data. We observe that the gaussian SVR provides the best prediction accuracy compared to other methods.

Table I summarises the values of the statistical scores obtained by the four models based on test data. Similar conclusions hold true and confirm that the Gaussian SVR 

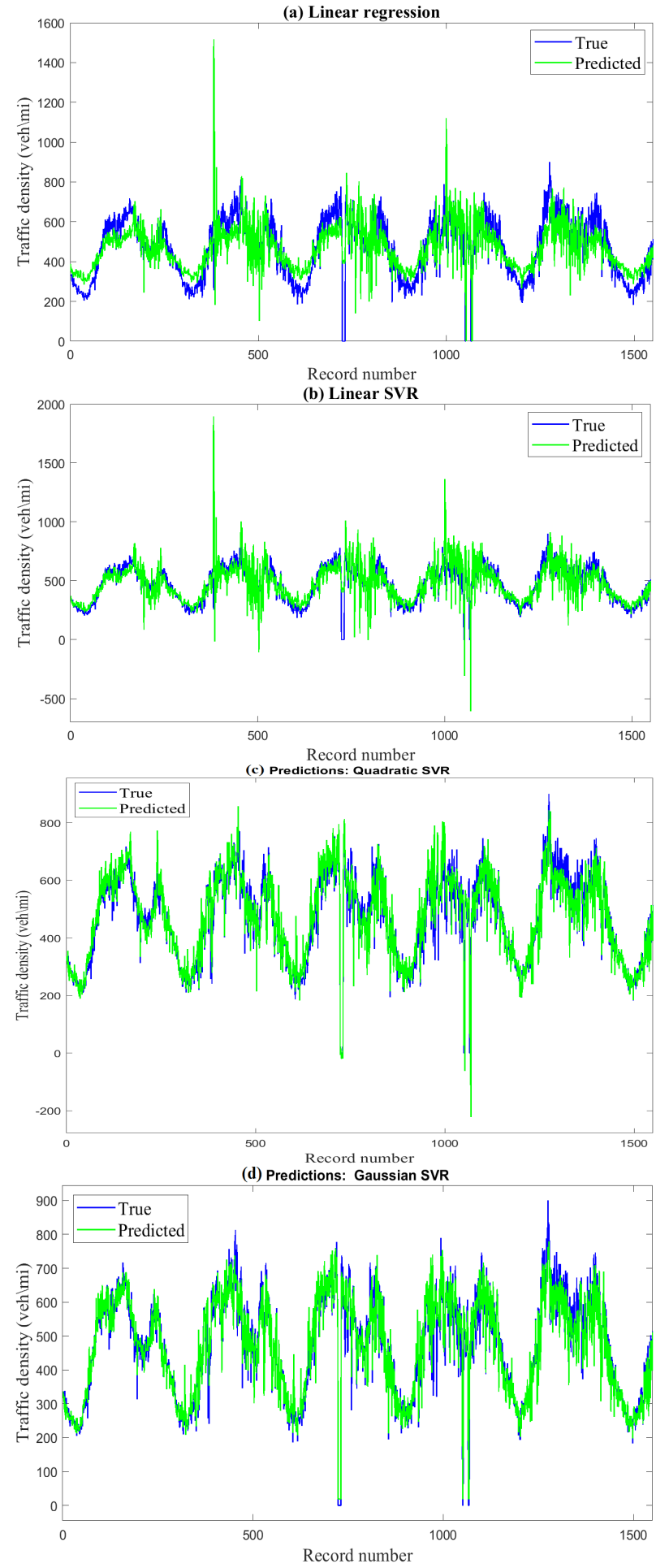

Fig. 4: Measured and predicted traffic flow by method using training dataset.

outperformed the other models by getting the lowest MAPE of 7.9\% followed by Quadratic SVR (8.5\%), Linear SVR $(14.6 \%)$, and linear regression (16.29\%). Overall, this study showed that kernel-based learning models, particularly Gaussian SVR, provide promising performance in capturing the
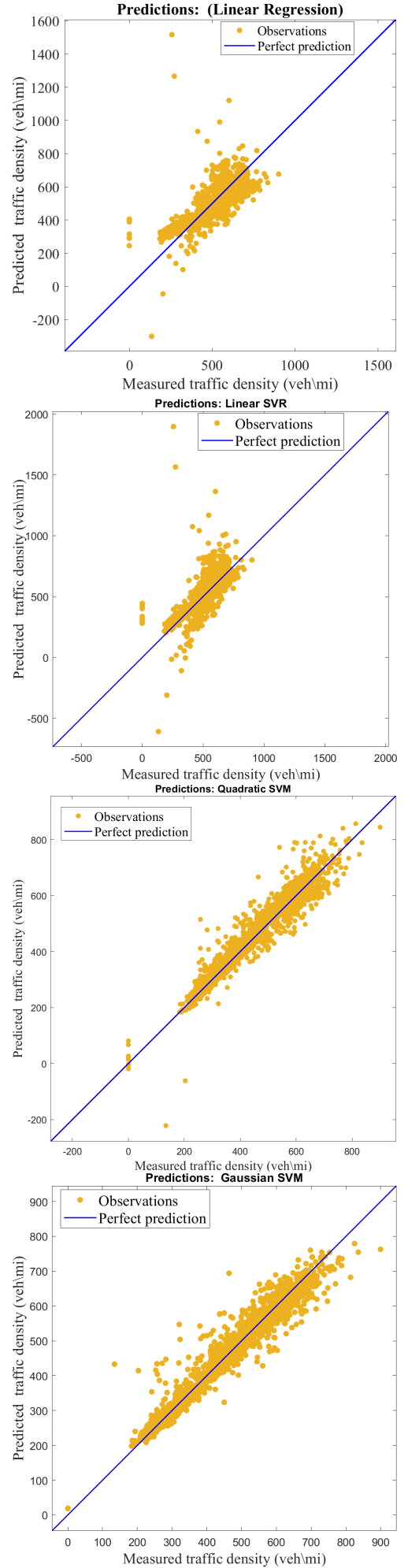

Fig. 5: Scatter graphs of measured and predicted traffic flow by method using training dataset.

nonlinearity features with high accuracy. 

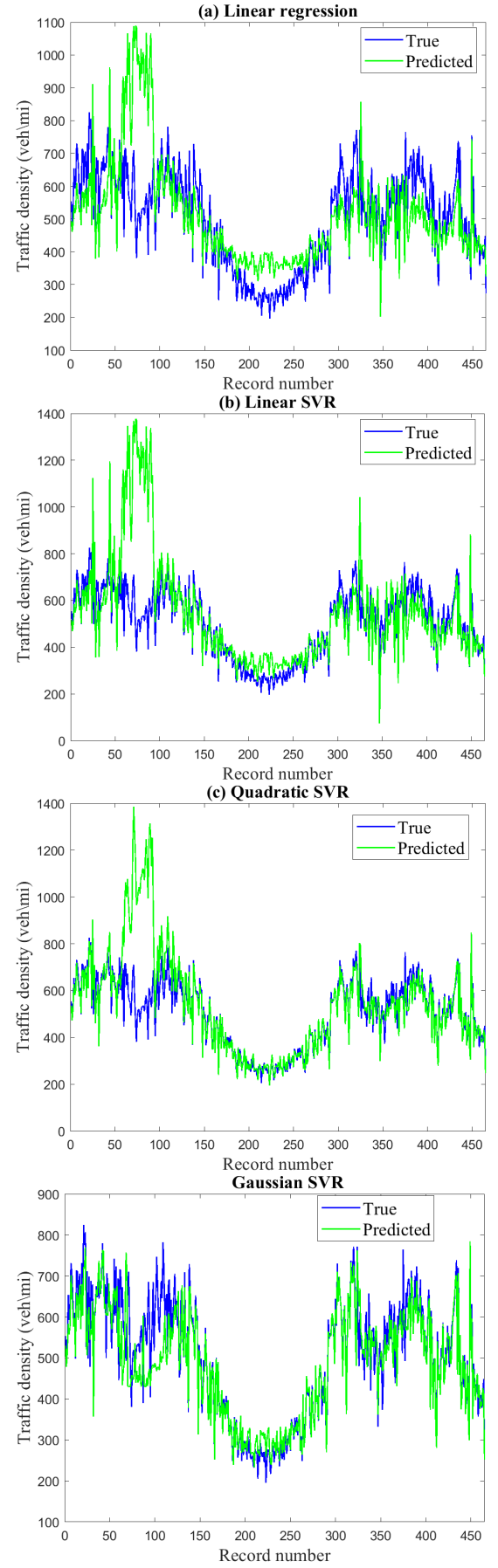

Fig. 6: Measured and predicted traffic flow by method using testing dataset.

TABLE II: Models evaluation based on RMSE, MAE and MAPE metrics.

\begin{tabular}{l|l|l|l}
\hline Models & RMSE & MAE & MAPE (\%) \\
\hline Linear Regression & 135.53 & 90.87 & 16.29 \\
Linear SVR & 187.067 & 95.16 & 14.60 \\
Quadratic SVR & 151.86 & 64.75 & 8.50 \\
Gaussian SVR & 62.74 & 38.23 & 7.90 \\
\hline
\end{tabular}

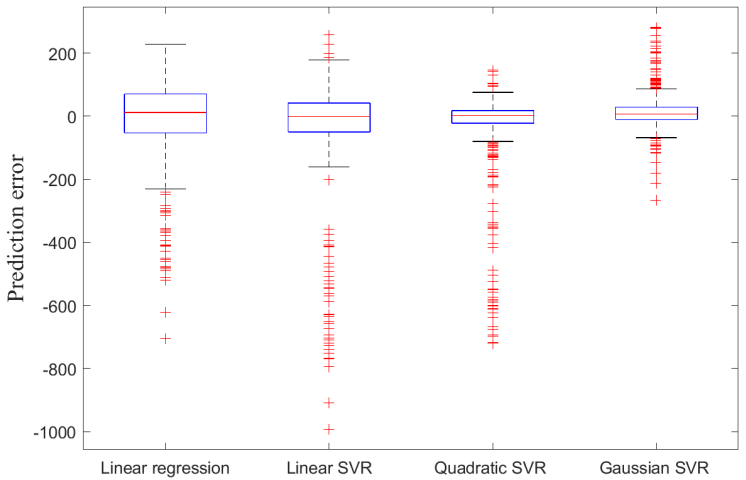

Fig. 7: Prediction errors by method from the testing dataset.

\section{CONClusion}

Accurate and reliable traffic flow prediction permits to avoid many transportation difficulties. It plays an essential role in ITS applications. In this paper, we presented a comparison between four kinds of regression methods, linear regression, linear SVR, quadratic SVR, and gaussian SVR. The results proved the superiority of the Gaussian SVR for traffic flow prediction. As future work, we plan to incorporate meteoro-

logical measurements and spatial information in constructing the machine learning models to further improve prediction quality. Thus, we plan to develop a more flexible prediction approach that considers spatiotemporal correlations of the traffic network and captures spatiotemporal features.

\section{ACKNOWLEDGMENT}

This publication is based upon work supported by King Abdullah University of Science and Technology (KAUST), Office of Sponsored Research (OSR) under Award No: OSR2019-CRG7-3800.

\section{REFERENCES}

[1] T. Afrin and N. Yodo, "A survey of road traffic congestion measures towards a sustainable and resilient transportation system," Sustainability, vol. 12 , no. 11 , p. 4660, 2020.

[2] A. Zeroual, F. Harrou, and Y. Sun, "Road traffic density estimation and congestion detection with a hybrid observer-based strategy," Sustainable cities and society, vol. 46, p. 101411, 2019.

[3] G. Li, W. Lai, X. Sui, X. Li, X. Qu, T. Zhang, and Y. Li, "Influence of traffic congestion on driver behavior in post-congestion driving," Accident Analysis \& Prevention, vol. 141, p. 105508, 2020.

[4] F. Harrou, A. Zeroual, and Y. Sun, "Traffic congestion monitoring using an improved knn strategy," Measurement, vol. 156, p. 107534, 2020.

[5] A. Haydari and Y. Yilmaz, "Deep reinforcement learning for intelligent transportation systems: A survey," IEEE Transactions on Intelligent Transportation Systems, 2020.

[6] Y. Cong, J. Wang, and X. Li, "Traffic flow forecasting by a least squares support vector machine with a fruit fly optimization algorithm," Procedia Engineering, vol. 137, pp. 59 - 68, 2016, green Intelligent Transportation System and Safety.

[7] T. Ouyang, X. Zha, L. Qin, Y. He, and Z. Tang, "Prediction of wind power ramp events based on residual correction," Renewable Energy, vol. 136, pp. 781 - 792, 2019.

[8] F. Harrou, A. Saidi, and Y. Sun, "Wind power prediction using bootstrap aggregating trees approach to enabling sustainable wind power integration in a smart grid," Energy Conversion and Management, vol. 201, p. 112077, 2019. 
[9] S. S. W. Bin Lu, Shaoquan Ni, "A support vector regression approach for investigating multianticipative driving behavior," Mathematical Problems in Engineering, 2015.

[10] Wei-Chiang Hong, Ping-Feng Pai, Shun-Lin Yang, and R. Theng, "Highway traffic forecasting by support vector regression model with tabu search algorithms," in The 2006 IEEE International Joint Conference on Neural Network Proceedings, July 2006, pp. 1617-1621.

[11] Z. H. Wei Ming, Yukun Bao and T. X. Multistep-Ahead, "Air passengers traffic prediction with hybrid arima-svms models." Hindawi Publishing Corporation Mathematial Problmes in Engineering, 2014.

[12] B. M. Williams and L. A. Hoel, "Modeling and forecasting vehicular traffic flow as a seasonal arima process: Theoretical basis and empirical results," Journal of Transportation Engineering, vol. 129, no. 6, pp. 664-672, 2003.

[13] M. G. Karlaftis and E. I. Vlahogianni, "Statistical methods versus neural networks in transportation research: Differences, similarities and some insights," Transportation Research Part C: Emerging Technologies, vol. 19, no. 3, pp. 387-399, 2011.

[14] W. Alajali, W. Zhou, S. Wen, and Y. Wang, "Intersection traffic prediction using decision tree models," Symmetry, vol. 10, no. 9, p. 386, 2018.

[15] S. Liu, H. Tai, Q. Ding, D. Li, L. Xu, and Y. Wei, "A hybrid approach of support vector regression with genetic algorithm optimization for aquaculture water quality prediction," Mathematical and Computer Modelling, vol. 58, no. 3, pp. 458 - 465, 2013, computer and Computing Technologies in Agriculture 2011 and Computer and Computing Technologies in Agriculture 2012.

[16] V. Vapnik, S. E. Golowich, and A. J. Smola, "Support vector method for function approximation, regression estimation and signal processing," in Advances in neural information processing systems, 1997, pp. 281-287.

[17] A. J. Smola and B. Schölkopf, "A tutorial on support vector regression," Statistics and computing, vol. 14, no. 3, pp. 199-222, 2004.

[18] Z. Yin and J. Hou, "Recent advances on SVM based fault diagnosis and process monitoring in complicated industrial processes," Neurocomputing, vol. 174, pp. 643-650, 2016.

[19] J. Lee, W. Wang, F. Harrou, and Y. Sun, "Reliable solar irradiance prediction using ensemble learning-based models: A comparative study," Energy Conversion and Management, vol. 208, p. 112582, 2020.

[20] J. Lee, W. Wang, F. Harrou, and Y. Sun,, "Wind power prediction using ensemble learning-based models," IEEE Access, vol. 8, pp. $61517-$ $61527,2020$.

[21] L. Xuan, L. Qian, J. Chen, X. Bai, and B. Wu, "State-of-charge prediction of battery management system based on principal component analysis and improved support vector machine for regression," IEEE Access, vol. 8, pp. $164693-164$ 704, 2020.

[22] B. Khaldi, F. Harrou, S. M. Benslimane, and Y. Sun, "A data-driven soft sensor for swarm motion speed prediction using ensemble learning methods," IEEE Sensors Journal, 2021.

[23] M. Awad and R. Khanna, Efficient learning machines: theories, concepts, and applications for engineers and system designers. Springer nature, 2015. 